\title{
SÍNDROME DE CAUDA EQUINA POST ANESTESIA RAQUIDEA EQUINE CAUDA SYNDROME POST ANESTESIA RAQUIDEA SÍNDROME DE EQUINA CAUDA POST ANESTESIA RAQUIDEA
}

\author{
Marcos Schioppi ${ }^{1}$ \\ ${ }^{1}$ Instituto de cardiología integral, Sanatorio CRAMI, Círculo Católico de Obreros del \\ Uruguay. mail: mschioppi@mail.com
}

\section{RESUMEN}

El uso de la anestesia regional se ha expandido en las últimas tres décadas, cada vez son más los profesionales que optan por esta técnica frente a la administración de anestesia general. La incidencia del síndrome de cauda equina SCE se reporta aproximadamente de 1 paciente en 32000. Se describe el caso de una paciente que presenta esta complicación, su tratamiento y evolución.

Palabras clave: Anestesia raquídea, síndrome de cauda equina, complicaciones.

\begin{abstract}
The use of regional anesthesia has expanded over the last three decades, more and more professionals opting for this technique versus the administration of general anesthesia. The incidence of SCE equine tail syndrome is reported in approximately 1 patient in 32000. The case of a patient presenting this complication, its treatment and evolution is described.
\end{abstract}

Key words: Spinal anesthesia, equine tail syndrome, complications. 
O uso da anestesia regional se expandiu nos últimos tres débitos, cada vez mais filho e os profesionales que optan por esta técnica frente a administração de anestesia geral. A incidencia da síndrome da cauda equina SCE se reporta a aproximadamente 1 paciente em 32000. Se descreve o caso de uma paciente que esta complicada, su tratamiento y evolución.

Palavras-chave: Anestesia raquídea, síndrome da cauda eqüina, complicações.

\section{INTRODUCCIÓN}

El uso de la anestesia regional se ha expandido en las últimas tres décadas, cada vez son más los profesionales que optan por esta técnica frente a la administración de anestesia general $^{(1)}$.

En nuestro medio se realizan cada año unas 150 mil anestesias, si bien no contamos con datos estadísticos nacionales podemos afirmar que los bloques nerviosos centrales ocupan un alto porcentaje de ellas, sus indicaciones han ido en aumento en el mundo ${ }^{(1,2)}$, abarcando procedimientos gineco obstétricos, traumatológicos, así como procedimientos urológicos y de cirugía vascular y general ${ }^{(3)}$ El anestésico local más utilizado en nuestro medio es bupivacaina al 0,5\% con el agregado de dextrosa lo que determina una solución hiperbárica. Es frecuente el agregado de opiáceos como coadyuvantes analgésicos, fentanil $50 \mathrm{mcg} / \mathrm{ml}$ y/o morfina al 1 por 1000, como complemento analgésico.

La incidencia de complicaciones de esta técnica ha disminuido notablemente en los últimos años, los avances en el conocimiento fisiopatológico, el advenimiento de 
fármacos con un perfil farmacocinética más seguro, así como las modificaciones realizadas en las agujas han permitido reducir dichas complicaciones ${ }^{(2,3)}$.

Dentro de las posibles complicaciones las neurológicas son las más frecuentes, seguida de la cefalea post punción y las infecciosas.

Afortunadamente muchas de estas complicaciones neurológicas tienen una evolución benigna -con o sin tratamiento- sin embargo, en algunos pacientes presentan daño neurológico irreversible. Estas conllevan un alto costo en salud y económico dado las secuelas que pueden sobrevenir (Tabla 1) ${ }^{(3)}$

Tabla 1:. Complicaciones asociadas a la anestesia neuroaxial.

\begin{tabular}{|l|c|c|c|}
\hline Causas de reclamo & Subaracnoidea & Peridural & Total \\
\hline Paro Cardiaco. & 2 & 0 & 2 \\
\hline Neurologicas. & 31 & 7 & 38 \\
\hline Infecciosas & 4 & 6 & 10 \\
\hline Toxixidad sistemica por AL & & 2 & 2 \\
\hline sobredosis de opioides & & 1 & 1 \\
\hline Cefaleas & 9 & 8 & 17 \\
\hline
\end{tabular}

La incidencia del síndrome de cauda equina SCE es baja, en un estudio de corte publicado en Suecia que incluyo un millón de pacientes encontró 32 casos, 1-32000 (4).

Aunque es poco frecuente SCE, se considera una complicación devastadora para el paciente y el técnico actuante. Este síndrome se define como el bloqueo sensitivo motor y vegetativo que permanencia más tiempo tiempos esperados para el tipo de bloqueo realizado y para los fármacos administrados. La evolución es poco predecible, pudiendo revertir espontáneamente en forma total o parcial en la mayoría de los casos, existiendo comunicaciones de casos con déficit neurológico permanente luego de años de seguimiento ${ }^{(3)}$ 
La primera complicación descripta de esta técnica fue la cefalea post punción dural, la misma fue descripta en el primer paciente en que se realizara la misma, fue 1898 cuando el Dr. August Karl Gustav Bier realizara por primera vez una anestesia raquídea.

En Gran Bretaña en 1987 se reportaron dos casos de paraplejia definitiva luego de recibir raquianestesia por el Dr. Malcolm Gram.

El diagnostico de esta entidad es clínico y su confirmación es imagenológica; el tratamiento debe ser instaurado en forma precoz, y se basa en la administración de corticoides y la fisioterapia intensa ${ }^{(3)}$

\section{CASO CLÍNICO}

Paciente de 52 años, sexo femenino, raza negra, coordinada para cirugía de hemorroides con dolor a nivel anal. Antecedentes de tabaquismo intenso desde la juventud, diagnóstico de bronquitis crónica, tratada con inhaladores en forma esporádica. Hipertensa (HTA) crónica tratada irregularmente con amlodipina, enalapril y diuréticos con regular control de cifras tensionales (refiere tener $160 \mathrm{mmHg}$. de presión sistólica en forma habitual). Diabetes Mellitus insulina-requirente en tratamiento con hipoglicemiantes orales, con un mal control metabólico (ocasionalmente cifras de 2 - 3 gr/dl). Sin historia de ángor ni disnea de esfuerzo, no elementos de macro o microangiopatía. Trombofilia diagnosticada 4 años previo a la cirugía por cuadro de oclusión arterial aguda humeral, tratada con ácido acetilsalicílico y Clopidogrel que se suspende 10 días antes de la cirugía.

Antecedentes anestésico-quirúrgicos de trombectomia y colocación de stent en arteria humeral con anestesia general sin incidentes. 
Al examen físico: sobrepeso (IMC=29), buena apertura bucal, Mallampati 1 y distancias de cuello normales. Ritmo cardiaco regular de 65 ciclos por minuto, sin soplos y presión arterial 155/90. Examen pleuropulmonar normal. Columna lumbar con buena palpación de los espacios interespinosos. Exámenes complementarios: Ecocardiograma normal. Se destaca hemoglobina glicocilada de $9 \mathrm{mg} / \mathrm{dl}$, glicemia de $1,6 \mathrm{gr} / \mathrm{dl}$. Resto de los exámenes complementarios: normales. Se discute con paciente y se decide realizar una técnica regional en silla de montar con monitorización estándar de la Sociedad Americana de Anestesia.

En posición de sedestación, se realiza punción raquídea a nivel L4 -L5 con aguja 27 G punta lápiz y se administran $7 \mathrm{mg}$ de bupivacaina hiperbárica al 0.5\%, 20 microgramos de fentanyl y 150 microgramos de morfina de uso espinal. La punción fue única, sin dolor ni parestesias Procedimiento sin incidentes. Luego de 5 minutos con la paciente sentada se ubica en posición de litotomía y se realiza la intervención quirúrgica (duración 45 min). Durante el procedimiento se mantienen cifras de normo-tensión y no se presentaron complicaciones. La paciente pasa a sala de recuperación postoperatoria y luego de dos horas se informa al anestesiólogo que la paciente no moviliza las piernas. Se constata bloqueo motor completo bilateral y bloqueo sensitivo a nivel de T10. Se realizan exámenes neurológicos seriados cada 60 minutos. A las 4 horas de la cirugía, moviliza el pie izquierdo en el plano de la cama sin reversión del bloqueo sensitivo.

Se hace consulta a neurocirujano con diagnostico presuntivo de hematoma espinal o de reacción inflamatoria local. Se coordina resonancia nuclear magnética (RNM) de urgencia que se logra realizar a las 12 horas y que se informa como normal. Se comienza tratamiento empírico con dosis de dexametasona de $12 \mathrm{mg}$ cada 8 horas y se consulta a endocrinólogo para normalizar las cifras de glicemia. Se coloca bomba de infusión continua de insulina ajustando según HGT. Los síntomas no revierten. 
Luego de 48 horas de tratamiento se cambia a 3 gramos de metilprednisolona en 24 horas por bomba de infusión continúa lográndose una mejoría de los síntomas a partir de las 12 de infusión. A las 24 horas la paciente moviliza ambas piernas venciendo la gravedad con su miembro inferior derecho, flexionando el miembro inferior izquierdo, se reduce la dosis de metilprednisolona a 2 gramos en 24 horas. Se realiza fisioterapia intensa y precoz. A las 72 horas la paciente logra ponerse de pie y a las 96 horas deambula con ayuda. A pesar de la mejoría motora y sensitiva no se recupera el control de los esfínteres. Diez días después la paciente presenta una complicación dolorosa de pierna derecha que fue interpretada inicialmente como una trombosis venosa profunda (TVP) pero no se confirma, encontrándose desgarro muscular en dicha pierna.

En el control alejado la paciente recobra control de esfínter anal a los 30 días y persistió con dificultades en la micción hasta seis meses después donde recupero totalmente la función de los esfínteres, quedando sin ninguna secuela.

\section{DISCUSIÓN}

La anestesia raquídea está ampliamente difundida en nuestro medio, su incidencia de complicaciones es baja lo que determina una falsa sensación de seguridad. Dentro de las complicaciones graves las neurológicas son las más frecuentes pudiendo producir lesiones severas e incapacitantes y determinan alto costo al sistema de salud. 3 La evidencia disponible proviene casi en su totalidad de reporte de casos clínicos sin embargo podrían presentar mayor riesgo de sufrir esta complicación, pacientes diabéticos con neuropatía demostrada, obesos, mayores de 50 años y posición de litotomía.3, 5, 6 
En nuestro medio el horario de la intervención quirúrgica lo fija el cirujano y no es infrecuente que se coordine al final de la jornada laboral independientemente del procedimiento o complejidad del paciente, esto deriva en un aumento del riesgo para los pacientes por varios motivos, desde la escasez del personal, la demora en los procedimientos diagnósticos hasta el cansancio de los médicos y técnicos.

El paciente quirúrgico habitualmente presenta distintos grados de ansiedad y estrés en el preoperatorio, sumado en nuestra paciente la abstinencia al tabaco, por estar ingresada en el sanatorio. Esto determina una respuesta simpática humoral con liberación de las hormonas de estrés y que seguramente explican las cifras elevadas de presión arterial y de glicemia que presentaba la paciente. Esta situación es frecuente en la práctica anestésica diaria y suele generar la duda de si se debe proceder o no con la cirugía. Ante el planteo de suspender el procedimiento, se evalúan los riesgos y beneficios de proceder o diferir la cirugía y se opta por continuar.

Luego de discutir las ventajas y desventajas de las diferentes técnicas anestésicas y se decidió realizar anestesia regional, Se realizó una anestesia raquídea en silla de montar, la misma cumplía con los requerimientos quirúrgicos, evita la manipulación de las vías respiratorios en una paciente EPOC, evita también la descarga adrenérgica de la laringoscopia y IOT, permitiendo a la vez monitorizar conciencia y minimizando los riesgos de una hipoglicemia intraoperatoria, en el contexto analizado consideramos era la técnica de elección.

Algunos autores plantean que el síndrome de cauda equina puede presentarse con mayor frecuencia en la posición de litotomía en particular en pacientes obesos donde el espacio subaracnoideo esta reducido pudiera contribuir en una mala distribución del anestésico con mayor riesgo de presentar aracnoiditis o poli radiculopatías. 5,6 
El síndrome de cauda equina se ha relaciona en el pasado con el uso de micro catéteres de uso intratecal y con concentraciones altas de anestésico local más que con su dosis, 2,7 en la actualidad donde el uso de lidocaína al $5 \%$ cayo en desuso la mayoría de los casos reportados hacen referencia a soluciones de bupivacaina hiperbárica llevando a pensar que se vinculan a la dextrosa que se adiciona a la solución para aumentar la baricidad, sin embargo, existen casos reportes de pacientes que presentaron un síndrome de cauda equina donde se administró bupivacaina isobáricas. ${ }^{(5)}$

Algunos autores plantean que esta complicación pudiera corresponder a la contaminación de la aguja con antisépticos, publicando dos casos de pacientes que luego de una anestesia raquídea fueron a la perdida irreversible de la función, el autor planteo como posible mecanismo la contaminación del anestésico ya que sumergía las ampollas en antisépticos. ${ }^{(3),(8)}$

Algunos autores plantean mayor neurotoxicidad de la clorhexidina alcohólica versus su predecesor el iodo povidona, esto no pudo demostrarse en un estudio realizado en ratas, dado su mejor perfil antiséptico la ASA recomienda el uso de la clorhexidina alcohólica previo a la realización de bloqueos nerviosos, pero para disminuir el riesgo de contaminación de la aguja recomienda esperar dos a tres minutos luego de su aplicación. En el caso de la paciente ella negaba presencia de síntomas neurológicos que hicieran pensar en una polineuropatía diabética (PNPD) sin embargo había sido estudiada por neurólogo y tenía en su domicilio un estudio eléctrico de miembros inferiores que documentaban una polineuropatía diabética moderada y bilateral, informe que no constaba en la historia clínica (HC) al momento de la cirugía y que la paciente no recordaba. En ocasiones los pacientes pueden negar, olvidar o minimizar sus patologías en el preoperatorio o incluso en policlínica por lo que contar con la HC completa es vital para poder realizar una valoración correcta. 
Un estudio plantea que la presencia de lesiones nerviosas degenerativas -perdida de revestimiento de las células de Schwann en bloqueos periféricos podría ser un factor de riesgo de presentar complicaciones nerviosas, el mecanismo podría ser neuro toxicidad por mayor exposición neuronal por falta del revestimiento axonal, otro mecanismo involucrado podría ser hipoperfusión medular por compromiso microvascular. ${ }^{(2)}$

Sin embargo, la presencia de PNP no es hasta la fecha una contraindicación de la anestesia raquídea y no predice la aparición de esta complicación, por lo que seguramente no nos hubiera cambiado la técnica anestésica. Algunos autores recomiendan frente a la presencia de déficit neurológico, contar con examen exhaustivo previo al procedimiento para evitar posibles reclamos posteriores. ${ }^{(3)}$

Frente a la persistencia del bloqueo luego de dos horas de la administración de la anestesia nos hizo pensar en la presencia de una complicación mecánica de técnica, de un hematoma peridural, más en una paciente que recibía anti agregantes plaquetarios previamente. ${ }^{(2)}$

El hematoma peridural es una complicación grave que de no resolverse en las primeras horas el daño neurológico es permanente ${ }^{(2)}$, lamentablemente en muchas de las instituciones del país en las que no se cuenta con servicio de resonador de urgencia, no se logra realizar el estudio hasta 12 horas después de detectada la complicación.

Afortunadamente nuestra paciente presento otra complicación que no requería resolución quirúrgica, de lo contrario la evolución hubiera sido otra, en este contexto y con esta limitante es una posibilidad que algunos colegas se replanteen la seguridad de realizar cirugías programadas con técnicas regionales, en horarios donde no se cuenta con los recursos necesarios para resolver las posibles complicaciones.

Con respecto a las dificultades que se presentan a diario en acceder a la historia clínica completa, en Uruguay estamos en un momento de transición donde parte la misma se 
encuentra en papel y parte se encuentra digitalizada lo que facilita los errores en la interpretación y los diagnósticos clínicos, llevando a valoraciones incompletas en el caso del preoperatorio. Otra situación frecuente es el hecho de no disponer de personal administrativo en archivos médicos por lo tanto no disponemos de $\mathrm{HC}$ anterior fuera de horario de oficina.

En relación al tratamiento instituido, los distintos casos reportes que hemos mencionado anteriormente hacen referencia a la fisioterapia temprana e intensa, en otros se hace mención a la utilización de corticoides por su efecto antinflamatorio no especificándose que corticoide ni que dosis se utilizó(3)

En el caso de la paciente fue valorada por fisioterapeuta la mañana siguiente a la aparición de la complicación, se comenzó con movilización pasiva de ambos miembros inferiores sin poder comenzar la rehabilitación intensa hasta que la paciente recobro movilidad de sus MMII.

Se comenzó en post operatorio inmediato con dosis altas de dexametasona sin efectos clínicamente relevantes, sin embargo, luego de la administración de metilprednisolona la paciente presento una rápida recuperación de la función sensitivo-motora por lo que pensamos que este sea pudiera ser más efectivo en el tratamiento de esta complicación. No hemos encontrado evidencia publicada referente a los beneficios de un corticoides sobre otro en las complicaciones neurológicas de la anestesia raquídea por lo que no es posible descartar que la mejora se debiera al tiempo transcurrido de los corticoides y no tanto al cambio del fármaco. Es necesario estudios originales controlados para poder avanzar en esta afirmación.

\section{CONCLUSIONES}


Hemos asistido a una paciente con una complicación grave de una anestesia regional, de la cual se desconocían factores de riesgo y que llevo una evolución lenta pero favorable con el uso de metilprednisolona intravenosa a dosis altas.

Del análisis de este caso clínico surgen una serie de fallas del sistema que exponen a pacientes a graves consecuencias médicas y expone a los profesionales a acciones legales. Esta situación es aún más grave porque en Uruguay como en varios países de Latinoamérica, no se cuenta con un consentimiento informado universal y claro. La dificultad en el acceso de la historia clínica y la necesidad de una historia electrónica accesible es evidentemente una de las barreras para evitar complicaciones. También el acceso a exámenes y estudios como en este caso la RNM, incrementan las oportunidades de detener la evolución de un evento adverso.

\section{Bibliografía:}

1. Alonso C, Sobrino J, Romero J. A, Vázquez A,. Ojea M, Cabadas R. Cuadro de afectación polirradicular tras anestesia intradural con bupivacaína para cirugía perianal. Rev. Soc. Esp. Dolor. 2002; 9:546-548.

2. Asenjo J F, Artukoglu F. Complicaciones neurológicas en anestesia regional. Rev. Chil. Anestesia. 2007(junio); 36: 103-111.

3.Víctor M, Whizar L, Flores-Carrillo, J C. Complicaciones Neurológicas de la Anestesia Neuroaxial. Anestesia en Mexico 2006; 18 (3): 133-144.

4.Sarifakioglu AB, Yemisci OU, Yalbuzdag SA, Ciftkaya PO, Cosar NS. Cauda equine syndrome after cesarean section. Am J Phys Med Rehabil 2013;92 (2):179-182.

5. Traore M, Diallo AD, Youssouf C, Cheick Oumar G, Samba Karim T, Jeannette T. Cauda Equina Syndrome and Profound Hearing Loss After Spinal Anesthesia with Isobaric Bupivacaine. Anesth Analg 2006; 102:1863-4.

6. Espinoza AM. Síntomas Neurológicos Transitorios Post. Anestesia Raquídea. Rev. Chil. Anest, 2009;38: 28-35-39.

7. Páez-Serralde F,Uribe-Velázquez H, Marrón-Peña M, Silva-Blas L. Eventos adversos neurológicos mayores secundarios a la anestesia neuroaxial. Revista Mexica de Anestesia. 2008; 31: S274-S281.

8. Kopp L, Jacob A, Hebl J. Regional Anesthesia in Patients With Preexisting Neurologic Disease Regional Anestesia and Pain Medicine. 2015; 40: 5. 
